\title{
Re-visiting the HLA dogma
}

\author{
Antoine Roux ${ }^{1,2}$ and Stijn E. Verleden ${ }^{3}$
}

Affiliations: ${ }^{1}$ Pneumology, Adult Cystic Fibrosis Center and Lung Transplantation Dept, Foch Hospital, Suresnes, France. ${ }^{2}$ Université Versailles-Saint-Quentin-en-Yvelines, Versailles, France. ${ }^{3}$ Leuven Lung Transplant Group, Dept of Chronic Diseases, Metabolism and Ageing (CHROMETA), KU Leuven, Leuven, Belgium.

Correspondence: Antoine Roux, Pneumology, Adult Cystic Fibrosis Center and Lung Transplantation Dept, Foch Hospital, 40 rue Worth, Suresnes, France. E-mail: a.rouxQhopital-foch.org

\section{@ERSpublications}

HLA-G may be protective in solid organ transplantation http://bit.ly/2ZjnPFy

Cite this article as: Roux A, Verleden SE. Re-visiting the HLA dogma. Eur Respir J 2019; 54: 1901440 [https://doi.org/10.1183/13993003.01440-2019].

Unravelling the intricacies of the human leukocyte antigen (HLA) system has been fundamental to our understanding of the immunobiological discrimination of self from non-self. In the context of solid organ transplantation, the allo-immune response is predominantly driven by a varying but inevitable degree of HLA mismatch (classically HLA class I and II molecules) between donor organs and the recipient host. In general, donor-recipient HLA mismatching tends to be maximal for solid organs where prospective HLA matching is not possible due to logistic reasons. This is true for lung transplantation, although rapid screening cellular and antigenic assays at the time of transplantation and thereafter can help detect, mitigate and manage the risks associated with donor-specific antibody production in the recipient. More recently, the newly developed epitope analysis is a significant step forward for ever finer precision deciphering of immunogenic mismatch-driven donor-specific HLA antibody production (DSA) [1] and graft and recipient outcomes [2].

The HLA-G molecule is a non-classical HLA molecule that is involved in the general down-regulation of the immune response. Its function is modulated by its expression level: modified by single nucleotide polymorphisms (SNPs) with overall polymorphism levels generally much lower than for classical HLA molecules, and membranous or soluble isoforms resulting from alternative splicing. Its role has been documented in the common miracle of pregnancy tolerance and increasingly in the therapeutic miracle of solid organ transplantation [3, 4]. Indeed, expression levels [5] or certain haplotypes [6] have been associated with differential graft outcome. However, there is still a lot of controversy regarding its role and impact in the context of lung transplantation as increased expression in tissue and higher systemic levels of HLA-G have been associated with better outcomes, while lower levels in the bronchoalveolar lavage (BAL) are associated with an increased risk of chronic lung allograft dysfunction (CLAD).

In an impressive lung transplant cohort published in this issue of the European Respiratory Journal, LAZARTE et al. [7] used a holistic, multi-dimensional approach incorporating clinical measures: blood, lung biopsy and BAL sampling; and DNA, mRNA and protein assessments using quantitative PCR, immunofluorescence and ELISA assays, respectively, for HLA-G to demonstrate a significant association between certain HLA-G SNP alleles with differential graft outcomes; most likely driven by the level of HLA-G expression in the allograft epithelium.

More specifically, multivariable analysis revealed donor SNP+3142 was associated with increased mortality, whereas five donor HLA-G SNP alleles were associated with reduced all-cause mortality and two donor 
recipient paired HLA-G SNPs were associated with CLAD-free survival. Rejection-free patients also had increased HLA-G expression in the airway epithelium compared to the leukocytes on the same biopsy.

In the BAL compartment, more counter-intuitive results show that soluble HLA-G was higher in patients who will later develop CLAD, but no association between HLA-G transcripts in the BAL and CLAD was observed.

Although these results are very intriguing and suggest an important role of HLA-G in post-transplant CLAD development and mortality, there are several key questions that remain to be answered before we can meaningfully incorporate these test assays into routine clinical practice decision-making. First, and as pointed out by the authors, the technical approaches used in this report only explore a part of the HLA-G system (two isoforms among seven; only monomeric and not polymeric forms). Secondly, the likely interaction of specific HLA-G SNPs with both certain classical HLA alleles and HLA mismatch scenarios was not within the scope of this analysis and this, as well as a deeper analysis incorporating acute (humoral or cellular) rejection episodes and specific DSA level dynamics over time and in different immunosuppression contexts, may reveal important links between certain HLA-G polymorphisms and currently used monitoring tools. Thirdly, better delineating the strength or otherwise of any specific HLA-G SNPs, their expression or protein levels with currently used diagnostic measures of allograft rejection risk may further explain the mortality and CLAD-free survival differences observed in this report and therefore increase the utility and acceptance of the findings presented. Indeed, the development and persistence of DSA is one of the most important risk factors for antibody-mediated rejection and chronic lung allograft dysfunction $[8,9]$ and there is already evidence suggesting that the presence of anti-HLA antibodies is associated with graft HLA-G expression [5].

Despite the limitations outlined above, these results remain interesting enough to suggest that donor recipient HLA-G matching may be a potentially useful approach for improving graft outcomes in the future. The enthusiasm of any such suggestion, however, should be tempered given the likely logistic constraints involved in going down such a path. Even if the impact of HLA-G SNP analysis on moderately increasing the risk of poor graft outcomes is confirmed in further prospective, multicentre studies, one should consider the counter-position of any such strategy. In particular, such a matching strategy, possibly in addition with DQ matching where the body of evidence is relatively stronger [10], could significantly delay access to donor grafts and therefore increase mortality on the transplant waiting list.

An intriguing finding in the context of heart and lung transplantation is the observed opposite effects of HLA-G donor-recipient matching regarding transplant outcomes of these two thoracic organs when assessed separately. Indeed, donor recipient pairing at SNP-201 was associated with a poor outcome after heart transplantation [11], but a positive outcome in lung transplantation, which the authors largely attributed to "environmental" differences as the lung allograft was more intimately connected to the external environment making it more prone to colonisation and/or infection. It would therefore be interesting in subsequent studies to compare the rate of colonisation and infection according to the donor and recipient HLA-G genotype in lung transplantation; this could provide further evidence for an important role of HLA-G in lung transplant patients, as microbial infection with bacteria (Pseudomonas aeruginosa) [12], respiratory viruses [13] or fungi (Aspergillus fumigatus) [14] are established risk factors for poorer long-term outcomes in these patients.

Further evidence of the mechanistic pathways involving HLA-G in the cascade from lung transplantation to CLAD and mortality are desperately needed, especially given the aforementioned discrepancy of HLA-G matching and likely specific function in heart and lung transplantation. With this in mind, DupIN et al. [15] have already demonstrated that the inhibition of T-cell alloreactivity in the bronchial epithelium is impaired via pathways that are restored by neutralisation of HLA-G. Moreover, a correlation between increased BAL transforming growth factor (TGF)-B levels and plasma soluble HLA-G expression has been demonstrated [5], with TGF-B being the classical pro-fibrotic growth factor which can induce epithelial to mesenchymal [16], but also mesothelial to mesenchymal transition [17], which is also implicated in pulmonary fibrosis [18].

This study has to be complimented for putting the donor back to in the equation when considering development of CLAD and long-term patient outcome, and for providing further evidence that some donors and recipients are more prone to poorer post-transplant outcomes. Indeed, there are few studies that focus on donor parameters influencing long-term outcome, as most studies solely focus on recipient characteristics. The fact that donor organ specifics are most often forgotten can be partly explained by the fact that most studies investigating the association between extended criteria donors and recipient outcomes fail to detect meaningful long-term differences [19, 20]. More recently, however, there is evidence that donor DR15 and DR7 expression have been associated with CLAD susceptibility [21], while shorter donor lung telomere length has also been associated with increased risk of CLAD [22]. 
This study therefore further leverages the growing evidence base that donor genetics should always be considered as an important factor in long term graft outcomes as, at the very least, they are likely to modulate the alloimmune response and graft wound healing process. Here, LAZARTE et al. [7] did focus on HLA-G SNPs, but there are many more polymorphisms to be considered for both immune system and tissue repair modulation [23]. Arguably, newer technological approaches for genome-wide analysis, bioinformatic assessment and machine learning algorithms [24], integrated with expanding horizons of seeing and thinking [25], may lead to an increasingly accurate global equation for predicting long term graft outcomes in the future. Finally, and more broadly, any deeper understanding that we can gain of the specific role of HLA-G in determining lung transplantation outcomes may one day be able to be extrapolated back to a much wider array of immunological and infectious disease disorders in general, and in respiratory medicine in particular, with a potentially much greater impact on population health.

Conflict of interest: None declared.

\section{Reference}

1 Walton DC, Cantwell L, Hiho S, et al. HLA class II Eplet mismatch predicts de novo DSA formation post lung transplant. Transpl Immunol 2018; 51: 73-75.

2 Snanoudj R, Kamar N, Cassuto E, et al. Epitope load identifies kidney transplant recipients at risk of allosensitization following minimization of immunosuppression. Kidney Int 2019; 95: 1471-1485.

3 Rebmann V, da Silva Nardi F, Wagner B, et al. HLA-G as a tolerogenic molecule in transplantation and pregnancy. J Immunol Res 2014; 2014: 297073.

4 Carosella ED, Rouas-Freiss N, Tronik-Le Roux D, et al. HLA-G: an immune checkpoint molecule. Adv Immunol 2015; 127: 33-144.

5 Brugiere O, Thabut G, Krawice-Radanne I, et al. Role of HLA-G as a predictive marker of low risk of chronic rejection in lung transplant recipients: a clinical prospective study. Am J Transplant 2015; 15: 461-471.

6 Di Cristofaro J, Reynaud-Gaubert M, Carlini F, et al. HLA-G*01:04 UTR3 recipient correlates with lower survival and higher frequency of chronic rejection after lung transplantation. Am J Transplant 2015; 15: 2413-2420.

7 Lazarte J, Ma J, Martinu T, et al. Donor human leukocyte antigen-G single nucleotide polymorphisms are associated with post-lung transplant mortality. Eur Respir J 2019; 54: 1802126.

8 Brugiere O, Roux A, Le Pavec J, et al. Role of C1q-binding anti-HLA antibodies as a predictor of lung allograft outcome. Eur Respir J 2018; 52: 1701898.

9 Verleden SE, Vanaudenaerde BM, Emonds MP, et al. Donor-specific and -nonspecific HLA antibodies and outcome post lung transplantation. Eur Respir J 2017; 50: 1701248.

10 Tikkanen JM, Singer LG, Kim SJ, et al. De novo DQ-donor-specific antibodies are associated with chronic lung allograft dysfunction after lung transplantation. Am J Respir Crit Care Med 2016; 194: 596-606.

11 Lazarte J, Goldraich L, Manlhiot C, et al. Human leukocyte antigen G single-nucleotide polymorphism-201 (CC-CC) donor-recipient genotype matching as a predictor of severe cardiac allograft vasculopathy. J Heart Lung Transplant 2016; 35: 1101-1107.

12 Gregson AL, Wang X, Weigt SS, et al. Interaction between Pseudomonas and CXC chemokines increases risk of bronchiolitis obliterans syndrome and death in lung transplantation. Am J Respir Crit Care Med 2013; 187: 518-526.

13 Allyn PR, Duffy EL, Humphries RM, et al. Graft loss and CLAD-onset is hastened by viral pneumonia after lung transplantation. Transplantation 2016; 100: 2424-2431.

14 Weigt SS, Copeland CAF, Derhovanessian A, et al. Colonization with small conidia Aspergillus species is associated with bronchiolitis obliterans syndrome: a two-center validation study. Am J Transplant 2013; 13: 919-927.

15 Dupin C, Lhuillier E, Letuve S, et al. Inhibition of T cell alloreactivity by bronchial epithelium is impaired in lung transplant recipients, through pathways involving TGF-beta, IL-10 and HLA-G. Transplantation 2017; 101: 2192-2199.

16 Borthwick LA, Parker SM, Brougham KA, et al. Epithelial to mesenchymal transition (EMT) and airway remodelling after human lung transplantation. Thorax 2009; 64: 770-777.

17 Sacreas A, von der Thusen JH, van den Bosch TPP, et al. The pleural mesothelium and transforming growth factor-betal pathways in restrictive allograft syndrome: a pre-clinical investigation. J Heart Lung Transplant 2019; 38: $570-579$.

18 Sibinska Z, Tian X, Korfei M, et al. Amplified canonical transforming growth factor-beta signalling via heat shock protein 90 in pulmonary fibrosis. Eur Respir J 2017; 49: 1501941.

19 Kotecha S, Hobson J, Fuller J, et al. Continued successful evolution of extended criteria donor lungs for transplantation. Ann Thorac Surg 2017; 104: 1702-1709.

20 Somers J, Ruttens D, Verleden SE, et al. A decade of extended-criteria lung donors in a single center: was it justified? Transpl Int 2015; 28: 170-179.

21 Haynes LD, Julliard WA, Mezrich JD, et al. Specific donor HLA-DR types correlate with altered susceptibility to development of chronic lung allograft dysfunction. Transplantation 2018; 102: 1132-1138.

22 Faust HE, Golden JA, Rajalingam R, et al. Short lung transplant donor telomere length is associated with decreased CLAD-free survival. Thorax 2017; 72: 1052-1054.

23 Royer PJ, Olivera-Botello G, Koutsokera A, et al. Chronic lung allograft dysfunction: a systematic review of mechanisms. Transplantation 2016; 100: 1803-1814.

24 Rajkomar A, Dean J, Kohane I. Machine learning in medicine. N Engl J Med 2019; 380: 1347-1358.

25 Dorfmuller P, Kotsimbos T. Lung allograft loss: naming helps seeing... and vice versa! Eur Respir J 2015; 46: $1242-1246$. 